\title{
Review of: "Prognostic factors for mortality in invasive pneumococcal disease in adult: a system review and meta-analysis"
}

\author{
Citrawati Wungu ${ }^{1}$ \\ 1 Universitas Airlangga
}

Potential competing interests: The author(s) declared that no potential competing interests exist.

The present meta-analysis studied about prognostic factors for mortality in invasive pneumococcal disease in adult. The authors are covering interesting topic which can benefit for practical use. However, there are some points that need to be revised:

Major comments:

1. Is there any previously related meta-analysis or systematic review regarding this? How it can be compared and how the present study gives novelty should also be described to make it more interesting

2. Of the keywords used were: "odds ratio" OR "relative risk" OR "hazard ratio". It it also measured RR and HR, why did the pooled study used OR only?

3. The study design included were not mentioned. If it included all types of observational studies, please specify in the table for characteristic study

4. Were included studies should provide OR? If OR is not provided on the primary studies did the authors count it by themselves? If the study included were all cohort, why did it use OR instead of RR? Did the authors get all the OR data or is there some conversion techniques used?

5. Provide Forest plot for the overall mortality rate from IPD

6. Why did the Forest plot used random-effect instead of fixed-effect model? For included studies with low heterogeneity, fixed effect models should be preferred

7. Statistical explanation is lacking, for example:

1. how to analyze the overall mortality rate

2. explain the reason of random or fixed effect model used

3. subgroup analysis

4. explain the analysis of risk factors (for example, extracting $\mathrm{OR}$ and $\mathrm{Cl}$ from each study and analyze it using generic inverse variance...)

5. Sensitivity analysis

6. Publication bias assessment (e.g.Egger's test, Funnel plot)

7. Significance with $p$ value

8. The discussion is still lacking some more explanation, especially regarding the influence of: alcohol abuse, nursing home, nosocomial infection, underlying chronic diseases, solid organ tumor, and 
immunosuppressed status to the increased mortality

Minor comments:

1. Some typos are present. For example:

1. In the title, it should be systematic review instead of system review

2. The prevalences are written as: ..... per $1,00,000$. It should be 100,000 or $1,000,000$

2. As the OR for septic shock is high, it should be highlighted in the abstract to prevent septic shock in IPD

3. CHAHL database is uncommon, please provide what does it stand for, or is it CINAHL?

4. Explain the interpretation of the NOQS score

5. Put boxes in the Prismaflow to make it neat

6. For prevalence study, the minimum number of participants should be included in the inclusion/exclusion criteria

7. Did the authors include years in the inclusion criteria? As the obtained studies were between $2000-2020$

8. The language for the included studies should also be provided in the table of characteristic study

9. Explain how additonal records identified through other sources comes from, as it is not mentioned in the text

10. Specify the reasons of full-text included in the Prismaflow

11. Analysis of pooled mortality in different regions (for example Asia and Europe) would be interesting

12. Put the abbreviations below the table of characteristic study: $\mathrm{CCI}, \mathrm{PSI}, \mathrm{CRP}$, etc

13. On Figure 4: Risk factor of community-acquired infection versus. There should be some words missing after the word versus 